This item was submitted to Loughborough's Research Repository by the author.

Items in Figshare are protected by copyright, with all rights reserved, unless otherwise indicated.

\title{
An automated low-cost condition monitoring system for quality control of automotive speedometers
}

PLEASE CITE THE PUBLISHED VERSION

PUBLISHER

Professional Engineering Publishing / @ IMECHE

VERSION

VoR (Version of Record)

LICENCE

CC BY-NC-ND 4.0

\section{REPOSITORY RECORD}

Al-Habaibeh, A., and Robert M. Parkin. 2019. "An Automated Low-cost Condition Monitoring System for Quality Control of Automotive Speedometers". figshare. https://hdl.handle.net/2134/4704. 
This item was submitted to Loughborough's Institutional Repository (https://dspace.lboro.ac.uk/) by the author and is made available under the following Creative Commons Licence conditions.

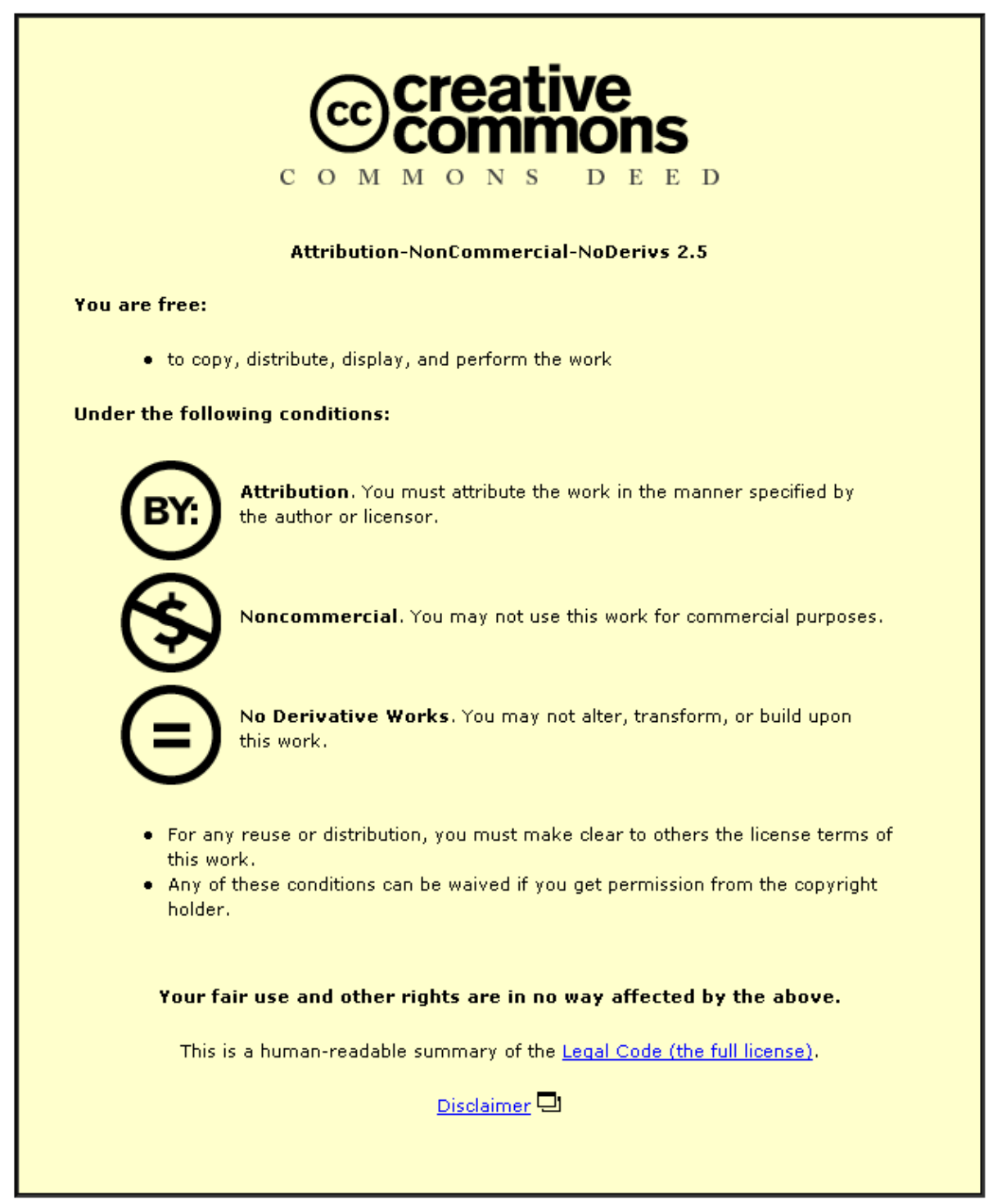

For the full text of this licence, please go to: http://creativecommons.org/licenses/by-nc-nd/2.5/ 


\title{
An automated low-cost condition monitoring system for quality control of automotive speedometers
}

\author{
A Al-Habaibeh* and R M Parkin \\ Mechatronics Research Centre, Wolfson School of Mechanical and Manufacturing Engineering, \\ Loughborough University, Leicestershire, UK
}

\begin{abstract}
The growing demands for high-quality products with improved specifications increase the requirements for condition monitoring and quality evaluation to ensure that no defective or belowspecification products reach the customer. This paper presents a low-cost condition-monitoring system using acoustics to evaluate the quality of mechanically driven automotive speedometers during manufacture. Signal conditioning methods employ discrete cosine transformation to simplify the frequency spectrum of the signals. A novelty detection approach is optimized to characterize the 'normal' conditions of the speedometers and to reject any speedometers that are not so categorized on the basis of the sound waves produced from experimental running of the speedometers. The suggested techniques are found useful in developing an automated condition-monitoring system for deselecting low-quality automotive speedometers.
\end{abstract}

Keywords: mechatronics, automotive, speedometers, condition monitoring, artificial intelligence, quality control

\section{NOTATION}

$p(\boldsymbol{x}) \quad$ unconditional probability density

$p\left(\boldsymbol{x} \mid C_{i}\right) \quad$ class-conditional probability density

$P\left(C_{i}\right) \quad$ prior probability

$P\left(C_{i} \mid \boldsymbol{x}\right)$ posterior probability

$\boldsymbol{x}$ data vector (time domain)

$X \quad$ data vector (frequency domain)

$\boldsymbol{y}(k)$ discrete cosine transformation vector of the $k$ th value

$\alpha_{j} \quad$ mixing coefficient (prior) of $\phi_{j}$

$\phi_{j} \quad$ response of the $j$ th Gaussian component

\section{INTRODUCTION}

Competition among car manufacturers and the increasing requirements of more comfortable modern cars has raised standards in terms of quality, reliability and

The MS was received on 31 July 2003 and was accepted after revision for publication on 2 September 2003.

*Corresponding author: Mechatronics Research Centre, Wolfson School of Mechanical and Manufacturing Engineering, Loughborough University, Loughborough, Leicestershire LE11 3TU, UK. performance. At the same time, the costs of today's cars are expected to be reduced due to the continuous drive of manufacturers for market share. In order to survive in such an environment, manufacturers need to develop sophisticated quality control and condition-monitoring systems in order to avoid any defective parts from reaching the consumer. The process of correcting faults once the car is assembled is time consuming and warranty rectifications after the vehicle has reached the customer are both expensive and damaging to a manufacturer's reputation.

The background cabin noise in road vehicles has been continuously reducing due to improvements in suspension, noise vibration and harshness, upholstery and insulation. The point has been reached where some of the 'carry-over' designs of speedometers from old models are now becoming too noisy to be used in modern vehicles without a proper condition-monitoring and quality control system during manufacture. One such design is the mechanical cable-driven speedometer. Despite its noise, it is still one of the popular and lowcost subsystems in some sectors of the car industry. However, these speedometers need to be tested before reaching the assembly line. Defective products have the potential to reach the final customer, which would damage the reputation of the company. Therefore, the main target is to have 'zero defects' for the monitoring strategy to be successful.

Proc. Instn Mech. Engrs Vol. 217 Part B: J. Engineering Manufacture 
The suggested monitoring strategy is to examine whether the tested devices are 'normal' or 'defective' via the measurement of the noise level. When a speedometer is found to be defective, the type of fault is detected and the problem (if consistent) is fed back to the manufacturing process for improvement.

The monitoring and inspection process could be performed by an operator. However, humans are often subjective and human errors could not be tolerated in a 'zero-defect' manufacturing environment. An automated methodology which could include a 'knowledge' database for quality control could also be implemented. The problems in using a human operator to perform the monitoring process can be summarized as follows:

(a) the variation in the hearing performance of different operators;

(b) the variation in an individual operator's hearing, during a shift and between shifts;

(c) background noise in the factory which could mask the speedometer noise;

(d) the inherent tendency of an operator to make human errors.

An automated inspection system is proposed in this paper. By automating the monitoring process, it is possible to remove human error and thus to increase efficiency and confidence in the output quality. A process which could identify defective speedometers and categorize them into defect classes would give some valuable feedback for the quality control strategy. The types of defect are those which cause unacceptable noise in the product. Although this could result in speedometers with other types of defect that have no apparent noise emission, other monitoring systems could be integrated with the proposed system to cover such faults.
This paper suggests the use of a low-cost microphone to monitor the quality of the produced speedometer using novelty detection. The literature available for the monitoring of speedometers is limited, which could be due to the commercial considerations. Most of the literature concerning automotive speedometers, such as reference [1], employs image processing methods for the inspection of systems. However, sound analysis has been reported for condition monitoring of a variety of systems such as machining operations [2], rolling element bearings [3], machinery in noisy environments [4] and fluid dynamics [5].

This paper reports a novel low-cost approach for automated monitoring system and inspection of automotive speedometers that can be performed in the factory to ensure non-defective products.

\section{AUTOMOTIVE SPEEDOMETERS}

\subsection{General structure and functionality}

A speedometer is an instrument that measures the speed at which the car is travelling and usually also embodies a mileage recording mechanism. It transforms the rotational movement generated by the car's gearbox into an angular deflection of a pointer (Fig. 1a). In mechanical versions, the rotational movement is supplied to the back of the speedometer via a flexible cable. This cable is connected to a magnetic shaft. The magnetic shaft spins a permanent magnet in the metal speed cup which is connected to the pointer. A spiral spring pulls the pointer towards the zero position where it rests against a pointer stop (when the magnetic shaft is stationary). The higher the speed of the vehicle, the

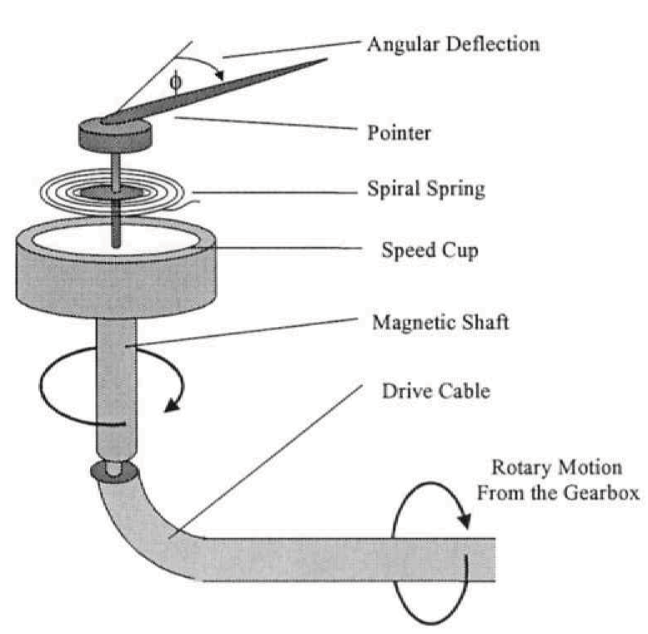

(a)

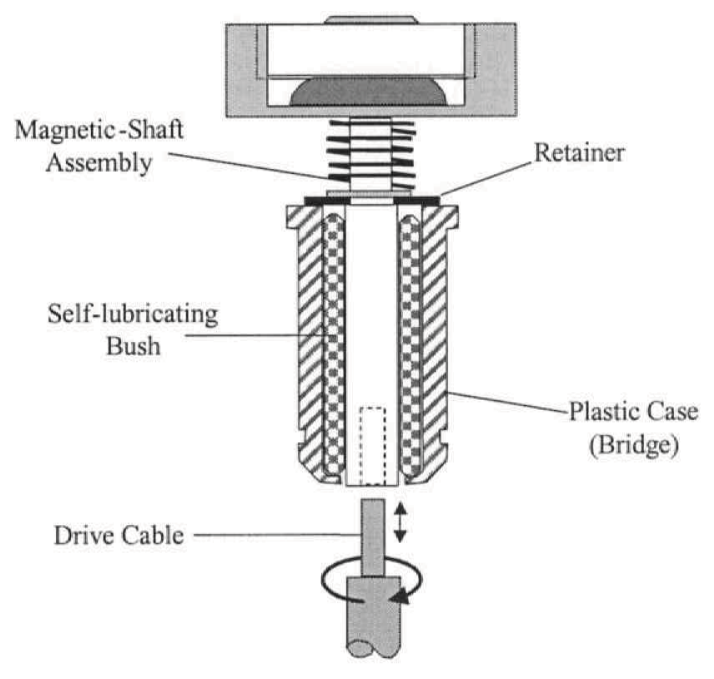

(b)

Fig. 1 (a) Schematic diagram showing the basic parts of the speedometer. (b) General arrangement of the speedometer bridge assembly 
higher is the speed of rotation of the magnet. The magnet rotates concentrically in the metal speed cup, in which the rotating magnetic field induces eddy currents which in turn produce a magnetic field of their own. The interaction of this magnetic field with that of the rotating permanent magnet exerts a torque on the speed cup. The faster the magnet rotates, the higher is the torque. The speed cup opposes the spring's torque and deflects the pointer. The deflected angle (shown as $\phi$ in the diagram) is directly proportional to the shaft speed.

\subsection{Possible manufacturing defects}

The speedometers are manufactured using a combination of manual and automated processes. Some of the typical defects, which can occur, are as follows:

1. Lack of grease. Although the magnetic shaft runs in a self-lubricating bush (see Fig. 1b), the shaft still requires grease to reduce noise and to minimize wear. If the grease is insufficient, the noise of the speedometer may be audible during certain driving conditions.

2. Parts out of tolerance. If the magnetic shaft assembly or self-lubricating bush is out of tolerance, excessive noise is heard by the driver.

3. Lack of watch oil. A lack of watch oil in the end of the magnetic shaft can cause the pointer of the speedometer to waver, but this does not cause an audible noise in most cases. However, the oscillatory pointer can be detected using an optical or visual system as described in reference [6].

\section{NOVELTY DETECTION}

Novelty detection [7] is a classification technique that recognizes presented data to be novel (i.e. new) or nonnovel (i.e. normal). The advantage of novelty detection comes from the ability to distinguish between training data and new data that have not been seen before. Different novelty detection algorithms and applications have been reported. Reference [8] discussed the extreme value theory and its application as novelty detection in biomedical data processing. In reference [9], generalized radial basis function neural networks are used to form a Bayesian classifier that is capable of detecting novel data. Reference [10] uses the novelty detection approach to diagnose failure in structures. In reference [11], novelty detection is used for the detection of special causes in multivariate statistical process control. The results of a literature review shows that novelty detection has the potential to be applied successfully in many applications.

The training data for the novelty detection algorithm consist of only one class (i.e. normal data), which is often much easier to obtain than data for multiple classes. Since a degree of overlap is normally expected between different classes, classification problems have a probabilistic nature [7]. Novelty detection involves estimating the probability density function (PDF) of a normal class from the training data and then estimating the probability that a new set of data belongs to the same class. The classification decision in novelty detection is based on Bayes' theorem as follows:

$$
P\left(C_{i} \mid \boldsymbol{x}\right)=\frac{p\left(\boldsymbol{x} \mid C_{i}\right) P\left(C_{i}\right)}{p(\boldsymbol{x})}
$$

where

$$
\begin{aligned}
P\left(C_{i} \mid \boldsymbol{x}\right)= & \text { probability that a given vector } \boldsymbol{x} \text { belongs to } \\
& \text { class } C_{i}
\end{aligned}
$$

For mutually exclusive classes containing all the data points, the unconditional probability density also satisfies the following equation:

$$
p(\boldsymbol{x})=\sum_{i=1}^{k} p\left(\boldsymbol{x} \mid C_{i}\right) P\left(C_{i}\right)
$$

where

$$
0 \leqslant P\left(C_{i}\right) \leqslant 1
$$

and

$$
\sum_{i=1}^{k} P\left(C_{i}\right)=1
$$

The accuracy of novelty detection classification is dependent on the accuracy of the modelled density functions [7]. Three main methods are used to model the PDF: parametric methods [12], non-parametric methods [13] and semi-parametric methods [14]. The parametric methods assume sufficient statistical information about the training data set that is not normally available. In non-parametric methods no assumptions are made regarding the underlying density functions and they depend on the training data to find the probability density function for a new input. Reference [7] classifies such methods as being kernel-based techniques and $K$ nearest-neighbour techniques. The $K$-nearest-neighbour method depends on the probability that $K$ number of 
data points of a vector fall within a specific volume. The kernel-based technique calculates the volume by defining width parameters for a number of known PDFs (kernels) to provide a general model for the training set. However, non-parametric methods require long computations for every input vector. Semiparametric density estimation is used in this paper for novelty detection because it combines the advantage of both parametric and non-parametric techniques and does not require extensive computational effort. Semiparametric methods use fewer kernels. A Gaussian mixture model is used in this paper to estimate the PDF. Unlike non-parametric methods the training data are used only during the process of construction of the density model and are not needed for calculation of the PDF for new vectors.

For a Gaussian mixture model, the following equation is used [7]:

$$
p(x)=\sum_{j=1}^{M} \phi_{j}(x) \alpha_{j}
$$

where

$$
\begin{aligned}
\phi_{j} & =\text { response of the } j \text { th Gaussian component } \\
\alpha_{j} & =\text { mixing coefficient (prior) of } \phi_{j}
\end{aligned}
$$

When the PDF is calculated, a threshold value can be imposed to define the borders between a novel vector and a normal data set [7]. Figure 2 explains the methodology through which the novelty detection works.

Novelty detection software NETLAB [15] is incorporated to investigate the acoustic signals obtained during the experimental work. More details regarding novelty detection can be found in references [7] and [15].

\section{SIGNAL PROCESSING AND SIMPLIFICATION METHODS}

For an automated monitoring strategy to be successful, it needs to operate in real time and on line. The training of novelty tends to be slow when relatively long vectors are used as input data. This is why many researchers expect a relatively high number of samples to be used for training the novelty algorithm. Also the information required to classify the signals is not always apparent in the time domain signals. Therefore, simplification and signal-processing methods are needed. Fast Fourier transformation [16] is used to transfer the sound waves from the time domain to the frequency domain. It is a signal-processing method that has been found useful in signal classification and condition monitoring [17]. A fast Fourier transform (FFT) is used to convert a digital signal $\boldsymbol{x}$ with length $N$ from the time domain into a signal $\boldsymbol{X}$ in the frequency domain:

$$
\boldsymbol{X}[h]=\sum_{i=0}^{N \times 1} \frac{1}{N} \boldsymbol{x}[i] W_{N} \quad \text { for } h=0,1,2, \ldots, N \times 1
$$

where $W_{N}=\mathrm{e}^{\times J 2 \pi \mathrm{i} h / N}$ and $J=\sqrt{\times 1}$.

In this research work, discrete cosine transformation [18-21] is used for data reduction by compressing the information contained in the harmonic signals. It is also useful to resample the signal and to reconstruct it from a partial number of the original values. The transformation of a signal to a discrete cosine transform produced a vector of the same length that contained the coefficients of the transformation.

\section{Input Vector}

$x$

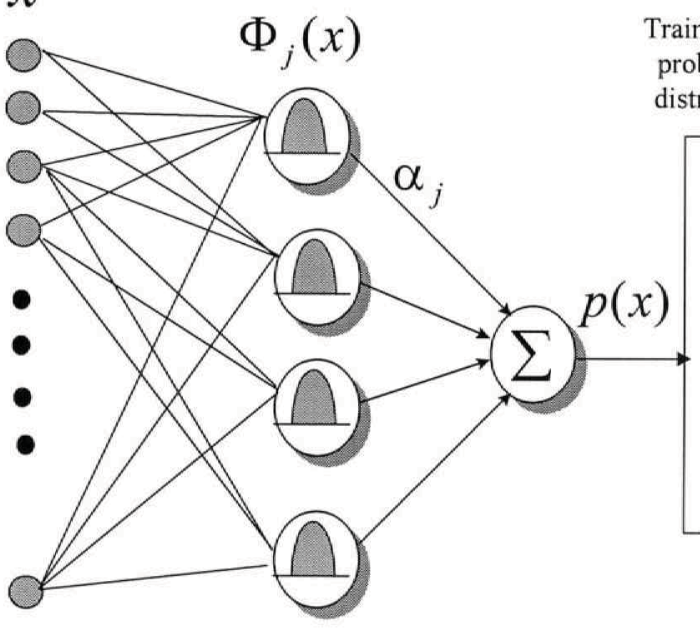

Threshold

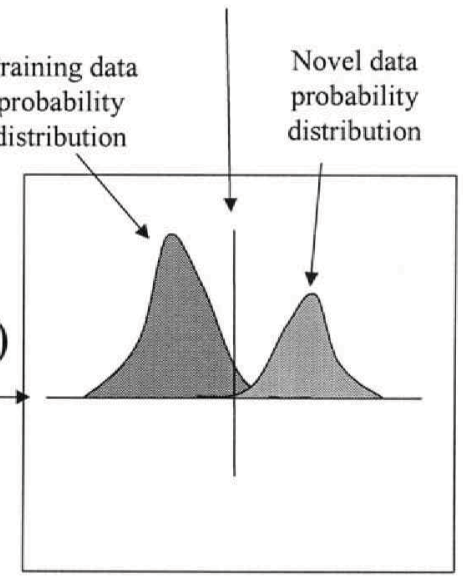

Fig. 2 The Gaussian mixture model and the way that it is used to detect novelty 
Mathematically, the discrete cosine transformation is defined as

$$
\begin{aligned}
\boldsymbol{y}(k)=\sum_{n=1}^{N} 2 \boldsymbol{x}(n) \cos \left[\frac{\pi}{2 N} k(2 n+1)\right], & \\
k & =0, \ldots, N \times 1
\end{aligned}
$$

The inverse discrete cosine transformation can be used to reconstruct an approximation of the original signal from the all discrete cosine transform coefficients or a partial number of the coefficients. The inverse discrete cosine transformation can be defined as [22]

$$
\begin{array}{r}
\boldsymbol{x}(n)=\frac{1}{N} \sum_{k=1}^{N} w(k) \boldsymbol{y}(k) \cos \left[\frac{\pi}{2 N} k(2 n+1)\right], \\
n=0, \ldots, N \times 1
\end{array}
$$

where

$$
w(k)= \begin{cases}\frac{1}{2}, & k=0 \\ 1, & 1 \leqslant k \leqslant N \times 1\end{cases}
$$

Discrete cosine transformation is used in this paper for data reduction to optimize and speed up the performance of the novelty detection algorithms. Although there are commonalities between discrete cosine transformation and fast Fourier transformation to retrieve the frequency spectrum of the signal, the fast Fourier transform is more convenient to use in industrial applications because many programming languages support fast Fourier transform functions.

\section{EXPERIMENTAL WORK}

The target of this research work is to develop a low-cost monitoring system for mechanically driven automotive speedometers that can be implemented on the shop floor of a manufacturing plant. By its nature, such an environment can be noisy and hence an automated monitoring system is needed. The selected set-up does not need to be compatible with a mass production environment and with low cost so that it can be implemented easily. The sensor to be used does not need to be in direct contact with the speedometers to ease the testing process. Therefore, a microphone was selected to measure the noise level via sound waves. In order to reduce the background noise the experiments were screened in a sound booth. A Brüel and Kjær condenser microphone type 4165 was used for this test connected to a 16 bit mono computer sound card to capture the sound waves. Special software was used to capture the digitized signals and to save them to the hard disk for further analysis. A sampling rate of 25000 samples/s was used to capture the signals. A total of 5020 samples/signal were acquired. A total of 24 different speedometers was used to test the system. These consist

SC03603 C IMechE 2003 of four different groups of six. The four groups are good speedometers, speedometers lacking watch oil, speedometers lacking grease on the magnetic shaft and speedometers with out-of-tolerance bushes. Six consecutive signals were recorded for each speedometer to increase the confidence in the detection process. It has been empirically determined that the maximum noise from the speedometers occurs at wheel speed of $40 \mathrm{~km} / \mathrm{h}$. Therefore, it was decided to use this speed to test the health of the speedometers.

\section{RESULTS AND DISCUSSION}

The training process for novelty detection was found to be slow and divergent when using the complete signals without the aid of signal-processing methods. This could be due to the limited number of training samples. Therefore, fast Fourier transform was used to extract the frequency information in the signals and a discrete cosine transform was used to simplify the data by reducing the number of samples. This made the features that characterize every type clearer. Figure 3 shows the effect of using a partial number of the coefficients to reconstruct the original frequency spectrum. It should be noted that less than 1 per cent of the samples was used to construct the same signal with sufficient detail.

The novelty detection NETLAB software [15] was used to analyse the acquired signals. The response $\phi_{j}$ of the Gaussian kernels [see Fig. 2 and equation (3)] is defined by a covariance matrix (a spherical matrix in this case) and a centre (i.e. the centroid of the input clusters). A single variance parameter for each Gaussian component was calculated using a different number of centres in the mixture model in order to select the most suitable structure based on the speed and the accuracy of results. This configuration implies that the model assumes that the input variables are all uncorrelated and independent, hence requiring only one variance parameter to be found for each Gaussian. More details have been described in reference [15]. Two healthy speedometers were used to define the 'normal' conditions. Six samples of every speedometer were used to define the normality. The rest of the samples were used to evaluate the novelty detection algorithm and to select the number of kernels and the length of the input vectors. Figure $4 \mathrm{a}$ shows the results obtained for different numbers of kernels and different dimensions of vectors. The training process for novelty detection was found to be slow and divergent for vectors that contain more than 140 elements. The logarithmic values are used to simplify the presentation of data. The $x$ axis presents the length of the input vectors (i.e. number of samples). The $y$ axis presents the difference between the minimum probability values of the normal data and the maximum probability values of the novel data. Therefore, the 

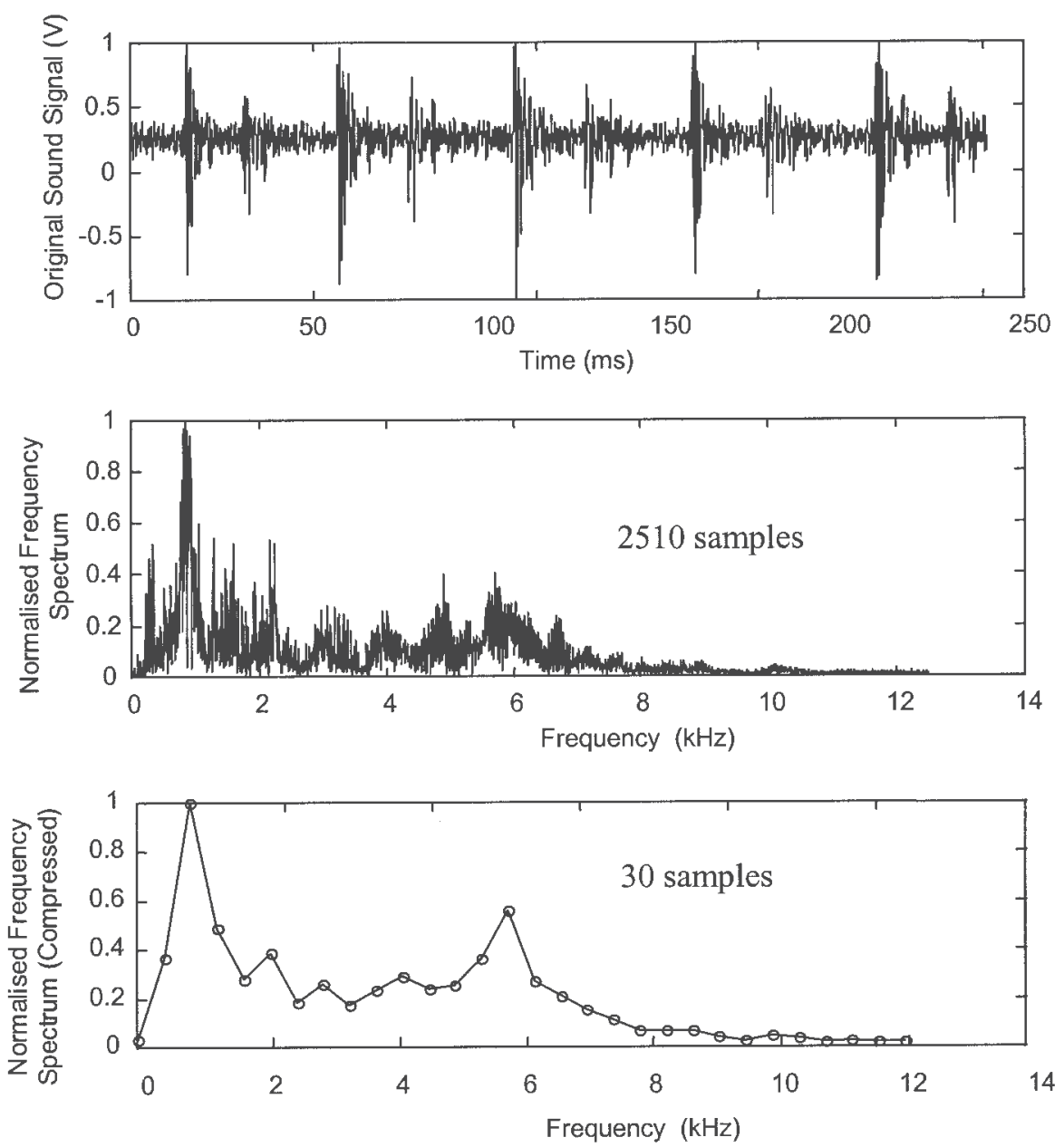

Fig. 3 The application of the discrete cosine transformation for data reduction of signals

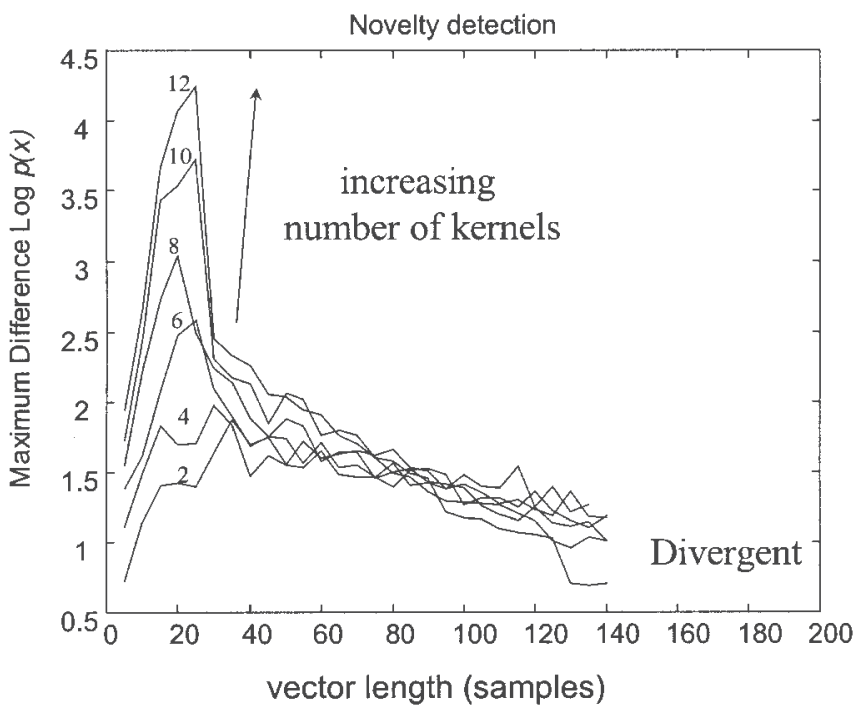

(a)

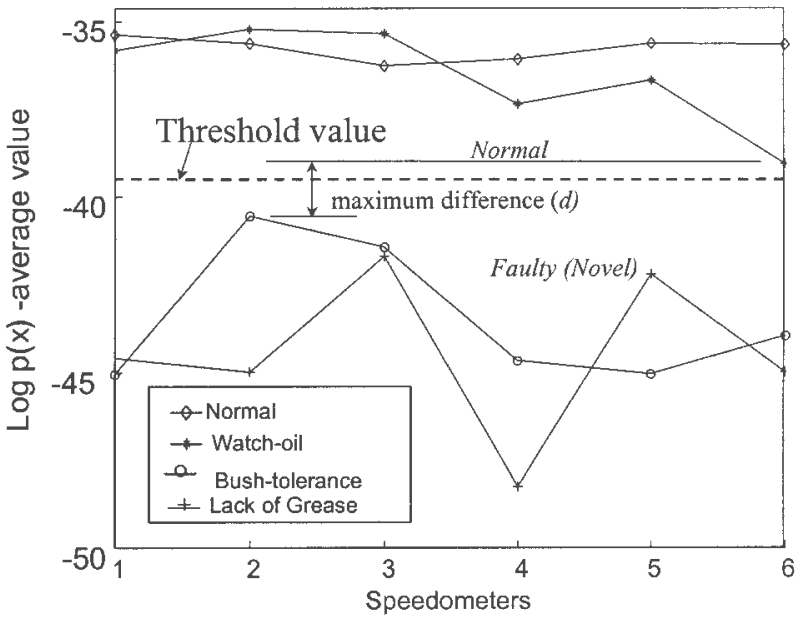

(b)

Fig. 4 (a) Optimization of the length of input vectors and number of kernels. (b) Novelty detection of 36 speedometers 
higher the difference, the more accurate the results are expected to be. It has been found that for all number of kernels the optimum number of length for the input data is between 20 and 30 inputs. Also the most accurate result is found for 12 kernels.

The average results of the tested speedometers for the 25 inputs and 12 kernels are shown in Fig. 4b. The maximum difference $d$, shown in Fig. 4b, is presented as the $y$ axis in Fig. 4a. The threshold value can be selected to achieve the required type-I and type-II errors (i.e. the error of rejecting a normal speedometer and the error of accepting a faulty speedometer respectively). As shown in Fig. 4b, the sound signals fail to detect the oscillation in the pointer of the speedometers due to lack of watch oil. However, the other two faults are clearly below the threshold value. In most cases, it is necessary to have a large number of training samples in order to accurately construct the PDF of the normal case. However, the processing of the original signals has made the features that differentiate between every type of speedometer clearer and has thus reduced the required number of samples to construct the PDF. The extraction of six sound signals from each speedometer was found useful to characterize normal and faulty speedometers. Theoretically, the difference between the signals from the same class is the presence of uncorrelated independent noise. The experimental work conducted has proven that it is necessary to acquire more than one signal since the behaviour of the speedometer could change during rotation. This has become clearer when monitoring faulty speedometers with intermittent faults. The lack of watch oil which causes the pointer to oscillate could be detected using an optical or an image analysis system, but not via the methods discussed in this paper.

\section{CONCLUSIONS AND FURTHER WORK}

The paper has presented a quality control problem which involves the monitoring of the noise level of mechanically driven automotive speedometers. A lowcost system using sound waves has been shown to be effective in detecting the quality of the automotive speedometers. A frequency spectrum of signals combined with a data reduction approach using discrete cosine transformation was used to simplify the information contained in the signals. A novelty detection approach was utilized to detect noisy speedometers from normal speedometers. Oscillatory pointers in some speedometers caused by the lack of watch oil would need to be detected using a visual means such as image processing or other optical techniques. Future work will consist in comparing the results obtained in this research work with the application of a principal component analysis. The authors will also be reporting on the implementation of the suggested

SC03603 (C) IMechE 2003 approach for detecting noise level combined with visual detection of oscillatory speedometers in the production line of a speedometer manufacturer.

\section{REFERENCES}

1 Elick, R. and Yoder, S. Automating speedometer inspections. Robotics World, June 1987, 5(6), 22-25.

2 Mannan, M. A., Kassim, A. and Jing, M. Application of image and sound analysis techniques to monitor the condition of cutting tools. Pattern Recognition Lett., October 2000, 21(11), 969-976.

3 Heng, R. B. W. and Nor, M. J. M. Statistical analysis and vibration signals for monitoring rolling element bearing condition. Appl. Acoust., January-March 1998, 53(1-3), 211-226.

4 Carney, M. S., Mann, J. A. and Gagliardi, J. Adaptive filtering of sound pressure signals for monitoring machinery in noisy environments. Appl. Acoust., 1994, 43(4), 333-351.

5 Belchamber, R. and Collins, M. Sound processing for your plant? Control Instrumentation, October 1993, 25(10), 4142.

6 Devaraj, G., Rajnarayanan, S. B. and Senthilnathan, A. Automating speedometer calibration. Evaluation Engng, 2000, 39(11), 110-131.

7 Bishop, C. M. Neural Networks for Pattern Recognition, 1995 (Clarendon, Oxford).

8 Roberts, S. J. Extreme value statistics for novelty detection in biomedical data processing. Proc. Instn Electl Engrs: Sci. Measmt Technol., November 2000, 147(6), 363-367.

9 Albrecht, S., Bush, J., Kloppenburg, M., Metze, F. and Tavan, P. Generalised radial basis function networks for classification and novelty detection: self-organisation of optimal Bayesian decision. Neural Networks, December 2000, 13(10), 1075-1093.

10 Manson, G., Pierce, S. G., Worden, K., Monnier, T., Guy, P. and Atherton, K. Long-term stability of normal condition data for novelty detection. Proc. SPIE, 2000, 3985, 323334.

11 Zorriassatine, F. Application of neural networks for detection of special causes in multivariate statistical process control. PhD thesis, University of Nottingham, April 2000.

12 Fukunaga, K. Introduction to Statistical Pattern Recognition, 2nd edition, 1990 (Academic Press, London).

13 Parzen, E. Stochastic Processes, 1962 (Holden-Day, San Francisco, California).

14 Specht, D. F. Probabilistic neural networks. Neural Networks, 1990, 3(1), 109-118.

15 Nabney, I. and Bishop, C. M. Netlab neural network software. http://www.ncrg.aston.ac.uk/netlab/index.html, Neural Computing Research Group, Information Engineering, Aston University, Birmingham B4 7ET, UK, October 2000.

16 Haykin, S. Neural Networks-A Comprehensive Foundation, 1999 (Prentice-Hall, Englewood Cliffs, New Jersey).

17 Demuth, H. and Beale, M. Neural Network Toolbox for Use with MATLAB, User's Guide, 1994 (The MathWorks Inc., Natick, Massachusetts).

Proc. Instn Mech. Engrs Vol. 217 Part B: J. Engineering Manufacture 
18 Pao, Y. H. Adaptive Pattern Recognition and Neural Networks, 1989 (Addison-Wesley, Reading, Massachusetts).

19 Kohonen, T. Self-Organization and Associative Memory, 3rd edition, 1989 (Springer, Berlin).

20 Newland, D. E. An Introduction to Random Vibrations, Spectral and Wavelet Analysis, 1993 (Longman, London).
21 Al-Habaibeh, A. and Gindy, N. A new approach for systematic design of condition monitoring systems for milling processes. J. Mater. Processing Technol., November 2000, 107(1-3), 243-251.

22 Jain, A. K. Fundamentals of Digital Image Processing, 1989 (Prentice-Hall, Englewood Cliffs, New Jersey). 